the K\&L scale. Patients with stage I according to standard radiography had no erosive process.

In DMFs OP $(100 \%$ and $78 \%, \mathrm{OR}=1.28,95 \%, \mathrm{Cl}[1.08-1.5], \mathrm{p}=0.02)$, SO (74\% and $11 \%, \mathrm{OR}=6.8,95 \%, \mathrm{Cl}$ [2.6-17.8], $\mathrm{p}<0,0001)$, subchondral cysts (SC) $(61 \%$ and $24 \%, \mathrm{OR}=2.5,95 \%, \mathrm{Cl}[1.3-4.82], \mathrm{p}=0.005)$ and subluxations $(43 \%$ and $14 \%, \mathrm{OR}=3.2,95 \%, \mathrm{Cl}[1.3-8.23], \mathrm{p}=0.01)$ were significantly more often found in patients with EOA. In PIPs SO (43\% AND 5\%, OR=8.04, $95 \%, \mathrm{Cl}[1.93-33.5], \mathrm{p}=0.0005)$, SC (52\% and $27 \%, \mathrm{OR}=1.93,95 \%, \mathrm{Cl}[0.1-$ 3.73 ], $p=0.045)$ and subluxations $(17 \%$ and $0 \%, p=0.01)$ were significantly more frequently detected in patients with EOA compared to the non-erosive group. According to the results of the AUSCAN questionnaire, a significantly greater severity of pain was found in patients with EOA $(65 \%)$ in comparison with the non-erosive (30\%) form of $\mathrm{HOA}(\mathrm{OR}=2.19,95 \%, \mathrm{Cl}$ [1.23-3.9], $\mathrm{p}=0.008$ ).

Conclusion: DIPs is most often affected in OA of interphalangeal joints, less often PIPs, the most common symptoms are JSN and OP. At EOA in addition to more frequent detection OP, cysts, SO, subluxations in DIPs, SO, cysts and subluxations in PIPs, there is also significantly more pronounced pain according to AUSCAN data, it can be concluded that EOA is more severe in comparison with the non-erosive form of HOA.

Disclosure of Interests: Danil Kudinsky: None declared, Ludmila Alekseeva Grant/research support from: Bayer, Alexander Smirnov: None declared, Alexander Volkov: None declared, Olga Alekseeva: None declared, Elena Taskina: None declared, Anastasiia Sukhinina: None declared

DOI: 10.1136/annrheumdis-2020-eular.5326

\section{AB1113 DIAGNOSTIC VALUE OF ANTI-SA AND ANTI-HN RNP K ANTIBODIES IN SERONEGATIVE RHEUMATOID ARTHRITIS}

\section{Volkava ${ }^{1}$, A. Kundzer ${ }^{1} .{ }^{1}$ BelMAPGE, Minsk, Belarus}

Background: The diagnostics of seronegative rheumatoid arthritis (RA) remains the important issue in modern rheumatology. Other biomarkers are studied to close the diagnostic gap.

Objectives: The aim of work was to assess anti-Sa and anti-hnRNP K in patients with RA and to evaluate their diagnostic value in seronegative by anti-CCP and RF RA.

Methods: In the study were included 270 patients, which fulfill EULAR/ACR 2010 criteria for RA and 50 healthy controls. The mean of patients age was 52.80 (95\% $\mathrm{Cl}: 51.70-54.80$ years).

Levels of antibodies to anti-CCP and anti-Sa were evaluated by ELISA according to the instructions of the manufacturer (Euroimmune, Germany), anti-hnRNP K according to the instructions of the manufacturer ((Medipan, Germany). Levels of rheumatoid factor (RF) were assessed by kinetic nephelometry using an automatic analyzer Beckman Coulter (USA).

Results: Anti-CCP in patients were found 209 (77.41\%) patients, RF - in 192 (71.11\%) patients, anti-Sa - in $163(60.37 \%)$ patients, anti-hnRNP K - in 63 (23.33\%) patients. The incidence of Anti-CCP, RF, anti-Sa, anti-hnRNP K in RA was significantly higher $(\mathrm{p}<0.05)$ than in healthy individuals $(0(0 \%), 0(0 \%), 1$ (2.00\%), 0 (0\%) respectively).

The diagnostic sensitivity and specificity of anti-Sa to discriminate seronegative by anti-CCP and RF RA patients from healthy controls by ROC - analysis were determined and made up for anti-Sa - 50,00\% (95\%Cl: $26.1-73.9)$ and $96.77 \%$ (95\%Cl: 83.2 - 99.5) respectively (area under ROC-curve $0.734 ; 95 \% \mathrm{Cl}: 0.588-$ $0.850, p=0.0026)$, for anti-hnRNP K - 55,56\% (95\%Cl:35,3 - 74,5), 100,00\% $(95 \% \mathrm{Cl}: 95,7$ - 100,0) respectively (area under ROC-curve 0.778; 95\%Cl: $0.689-$ $0.851, p=0.0001$ ). There were no significant differences in clinical (DAS 28, CDAI, SDAI, treatment options) and laboratory characteristics (ESR, CRP, anti-CCP, RF levels) between anti-Sa-positive and anti-Sa-negative patients ( $p>0.05)$ as well as between anti-hnRNP K-positive and anti-hnRNP K-negative patients ( $p>0.05)$.

Conclusion: Anti-Sa and anti-hnRNP K may serve as potential additional laboratory biomarkers for seronegative RA to allow to close the seronegative RA gap and confirm the diagnosis of RA.

Disclosure of Interests: None declared

DOI: 10.1136/annrheumdis-2020-eular.5407

\section{$\mathrm{AB} 1114$ \\ HANDWORK VS MACHINE; A COMPARISON OF RHEUMATOID ARTHRITIS PATIENT POPULATIONS AS IDENTIFIED BY MACHINE-LEARNING AND CRITERIA- BASED CHART REVIEW.}

T. Maarseveen ${ }^{1}$, M. Maurits ${ }^{1}$, E. Niemantsverdriet ${ }^{1}$, T. Huizinga ${ }^{1}$, A. Van der Helm - van Mil ${ }^{1}$, R. Knevel ${ }^{1,2}{ }^{1}$ Leiden University Medical Center (LUMC),
Rheumatology, Leiden, Netherlands; ${ }^{2}$ Brigham and Women's Hospital, Rheumatology, Boston, United States of America

Background: Electronic Medical Records (EMRs) offer a magnitude of observational data which is currently often manually reviewed to identify patient cases. Machine-Learning (ML) methods are highly efficient tools for data extraction, being able to process the information-rich free-text physician notes from EMRs. [1]

We postulate that incorporating this data into an ML pipeline, will enable the high-throughput identification of RA case cohorts qualitatively equal to conventional EULAR/ACR criteria based chart review.

Objectives: To investigate the comparability of Rheumatoid arthritis (RA) cases identified through EMR free-text enriched machine-learning algorithms to the field's gold-standard (GS) of criteria based chart-review.

Methods: We developed a classification algorithm using a Support Vector Machine (SVM) trained on 1000 manually reviewed patient EMRs and validated on a separate 1000 records. [2]

The SVM assigns a probability of being an RA case to each patient at a speed of $>5000$ records per second. The probability cutoff for case identification can be tailored to studies' needs, optimizing on sensitivity, specificity, etc. We optimized on PPV, resulting in a cutoff of 0.48 with a PPV of 0.96 .

We used the 1987 and 2010 EULAR/ACR criteria based diagnoses of RA at one year after inclusion in a prospective arthritis cohort as GSs and compared the baseline characteristics of ML identified RA cases with both GSs separately and for those fulfilling at least one set of criteria using Pearson chi-squared and Mann-Whitney $U$ tests $(a=0.05)$.

Results: At the 0.48 cutoff the ML model performs very well on the annotated set and when compared to criteria based GSs. (Table 1)

Table 1. Performance of ML-model in the annotated data ${ }^{\dagger}(N=1000)$ predicting cases and 1987, 2010 or either criteria based data * $(N=1235$, 1218 \& 1244 respectively) predicting cases (prob. $\geq 0.48$ ). TP; True Positive, FP; False Positive, TN; True Negative, FN; False Negative, PPV; Positive Predictive Value, NPV; Negative Predictive Value, Spec; Specificity, Sens; Sensitivity

\begin{tabular}{llllll}
\hline & Accuracy & PPV & NPV & Spec & Sens \\
\hline Annotated Cases $^{\dagger}$ & 0.98 & 0.96 & 0.98 & 1.00 & 0.74 \\
Criteria Definite Cases 1987* $_{\text {Criteria Definite Cases 2010* }}^{*}$ & 0.82 & 0.79 & 0.83 & 0.92 & 0.61 \\
Either Criteria Cases* $^{*}$ & 0.78 & 0.81 & 0.77 & 0.93 & 0.55 \\
\hline
\end{tabular}

Since patients diagnosed with RA do not necessarily meet classification criteria it is not surprising that the ML cases and GSs do not completely overlap. The ML cases overlap to a larger degree with the 1987 GS than with the 2010 GS. Clinically, the ML identified cases do not differ from the 2010 and 1987 GS cohorts except for a slightly higher CCP2 positivity compared to the 1987 GS (65 vs $51 \%$ ) and the combined criteria (65 vs $56 \%$ ). (Table $1 \& 2$ )

Conclusion: ML algorithms processing clinician notes enable fast and efficient selection of cases that are clinically similar to cases selected by criteria based chart review. This allows a significant reduction of time and effort required to construct high quality research cohorts.

Table 2. Comparison of baseline characteristics between 3 ML defined cohorts and a criteria based gold standard. ${ }^{\dagger}$ Not statistically tested; * significantly different to the ML selected cases at $a=0.05$, Pearson ChiSquared for proportions, Mann-Whitney U for medians

Predicted 1987 Criteria 2010 Criteria Either Criteria Definite Case Based Case Based Case Based Case

$\mathrm{N}^{\dagger}$

Proportion Women

Proportion CCP2 Positive

Proportion RF positive

Median BMI

Median BSE

Median CRP

Median Age at Inclusion

Median Symptom Duration at Diagnosis (in Days)

Median Number of Swollen

Joints

$\begin{array}{cccc}335 & 399 & 609 & 666 \\ 0.67 & 0.63 & 0.65 & 0.65 \\ 0.65 & 0.51^{*} & 0.61 & 0.56^{*} \\ 0.67 & 0.60 & 0.69 & 0.64 \\ 26.0 & 25.5 & 25.6 & 25.6 \\ 28 & 29 & 26 & 26 \\ 9.6 & 11 & 9 & 9 \\ 57.7 & 58.7 & 57.2 & 58.3 \\ 95.5 & 90 & 91 & 89 \\ 5 & 6 & 6 & 5\end{array}$

\section{References:}

[1] Maarseveen et al. ARD 2019;78

[2] https://github.com/levrex/DiagnosisExtraction_ML

Acknowledgments: T. Maarseveen and M. Maurits contributed equally. 
Disclosure of Interests: Tjardo Maarseveen: None declared, Marc Maurits: None declared, Ellis Niemantsverdriet: None declared, Thomas Huizinga Grant/ research support from: Ablynx, Bristol-Myers Squibb, Roche, Sanofi, Consultant of: Ablynx, Bristol-Myers Squibb, Roche, Sanofi, Annette van der Helm - van Mil: None declared, Rachel Knevel: None declared

DOI: 10.1136/annrheumdis-2020-eular.5647

\section{AB1115 REAL-WORLD NAILFOLD VIDEOCAPILLAROSCOPY IN A REFERRAL CENTER IN NORTHWESTERN COLOMBIA: A RETROSPECTIVE COHORT STUDY}

D. C. Varela Tabares ${ }^{1}$, J. Gutiérrez Bolaños ${ }^{1}$, L. M. Rodríguez Padilla ${ }^{2}$, M. A. Mesa Navas ${ }^{3,4}$, C. J. Velásquez-Franco ${ }^{3,4}$ on behalf of EULAR Study Group on Microcirculation in Rheumatic Diseases.. UNIR Research Group. School of Health Sciences. Universidad Pontificia Bolivariana. Medellin, Colombia

${ }^{1}$ Universidad Pontificia Bolivariana, Internal Medicine, Medellin, Colombia;

${ }^{2}$ Universidad Pontificia Bolivariana, Research, Medellin, Colombia;

${ }^{3}$ Universidad Pontificia Bolivariana. Clinica Universitaria Bolivariana, Rheumatology, Medellin, Colombia; ${ }^{4}$ EULAR Study Group on Microcirculation in Rheumatic Diseases, Medellin, Colombia

Background: Nailfold videocapillaroscopy is a non-invasive tool for the assessment of peripheral microcirculation, and it is useful for the diagnosis and prognosis of systemic autoimmune diseases. Despite its frequent use in clinical practice, the indications of this procedure are not standardized and there is no clear information in real-life about the reasons for remission, the presence of clinical findings of autoimmune diseases during the procedure, the frequency of patterns of autoantibodies and specific capillaroscopic findings.

Objectives: To describe the sociodemographic, clinical, paraclinical, and capillaroscopic findings of a cohort of subjects referred to a capillaroscopy service in northwestern Colombia.

Methods: We conducted a retrospective cohort study, including subjects from 2015 to 2018. Patients were evaluated by two expert rheumatologists. Variables: Reasons for referral, capillaroscopic patterns at baseline and at 6-month follow-up, presence of clinical findings of systemic autoimmune diseases during the procedure (Raynaud's phenomenon, puffy fingers, sclerodactyly, pitting scars, digital ulcers, sclerosis cutis, platysma sign, Gottron, and microstomy), along with the pattern and dilution of antinuclear antibodies. Categorical variables were expressed in frequency and percentage and quantitative variables in mean and standard deviation or median with interquartile range, depending on the distribution of the data. Statistical package: SPSS 25. This survey was approved by the institutional Ethics Committee.

Results: A total of 392 capillaroscopies were performed, 318 for the first time. The referral reasons for capillaroscopy were: Raynaud's phenomenon ( $n=134 ; 42.1 \%)$, connective tissue disease different than systemic sclerosis (SSc) ( $n=105 ; 33.1 \%)$, and systemic sclerosis $(n=79 ; 24.8 \%)$. The baseline capillaroscopic patterns found were: Normal $(n=123 ; 38.7 \%)$, non-specific $(n=81 ; 25.5 \%)$, SSc $(n=90 ; 28.3 \%)$, scleroderma-like ( $n=24 ; 7.5 \%)$. Among SSc pattern, early $(21 / 90 ; 23.3 \%)$, active, $(38 / 90 ; 42.2 \%)$, and late patterns $(31 / 90 ; 34.4 \%)$ were found. Of the 12 capillaroscopies that presented a non-specific pattern at 6-month follow-up, only one (8.3\%) progressed to a systemic sclerosis pattern. In the SSc patterns, the frequency of clinical findings were: sclerodactyly ( $n=34 ; 37.8 \%)$, Raynaud`s phenomenon $(n=26$; $28.9 \%)$, puffy fingers $(n=10 ; 11.1 \%)$, platysma sign $(n=10 ; 11.1 \%)$, pitting scars $(n=8 ; 8.9 \%)$, digital ulcers $(n=8 ; 8.9 \%)$, telangiectasia $(n=7 ; 7.8 \%)$, microstomy $(n=4 ; 4.4 \%)$, and Gottron $(n=1 ; 1.1 \%)$. In the SSc patterns, $42 / 44$ subjects $(95,4 \%)$ had positive antinuclear antibodies in an mean dilution of $1: 320$; the most frequent patterns were: centromere $(n=27 ; 64.3 \%)$ and nucleolar $(n=6 ; 14-3 \%)$.

Conclusion: In a real-world setting, the main referral reason to a capillaroscopic center was Raynaud's phenomenon; more than a third of the subjects had normal capillaroscopic findings; in the subgroup of baseline non-specific pattern, most of them were normal during follow-up; sclerodactyly and Raynaud's phenomenon were the most frequent clinical findings in patients with systemic sclerosis capillaroscopic patterns.

References:

[1] Melchor S, et al. Semin Arthritis Rheum. 2016; 46(3): 350-5.

[2] Fichel F, et al. Dermatology. 2014;228(4): 360-7

Acknowledgments: School of Health Sciences. Universidad Pontificia Bolivariana. Clinica Universitaria Bolivariana. Medellin, Colombia

Disclosure of Interests: None declared

DOI: 10.1136/annrheumdis-2020-eular.1436

\section{AB1116 DOPPLER EVALUATION OF ENTHESITIS SEEMS TO BE A RELEVANT OUTCOME IN THE ASSESSMENT OF ACTIVITY IN SPONDYLOARTHRITIS AND PSORIATIC ARTHRITIS}

J. Molina Collada ${ }^{1}$, C. Macía-Villa ${ }^{2}$, C. Plasencia ${ }^{3}$, J. M. Alvaro-Gracia ${ }^{1}$, E. De Miguel ${ }^{3} .{ }^{1}$ Hospital Universitario Gregorio Marañón, Madrid, Spain; ${ }^{2}$ Hospital
Universitario Severo Ochoa, Madrid, Spain; ${ }^{3}$ Hospital Universitario La Paz, Madrid, Spain

Background: The assessment of activity in spondyloarthritis (SpA) and psoriatic arthritis (PsA) involves several domains, including enthesitis. Clinical enthesitis evaluation has shown low sensitivity, specificity and reliability. Ultrasound (US) examination of enthesitis can be an accurate and objective way to evaluate this domain, supporting its inclusion in the assessment of the global state of the disease

Objectives: The main objective of this study is to analyze de prevalence of Doppler enthesitis in active SpA and PsA patients and to evaluate its association with the disease activity at patient level prior to start a biological therapy

Methods: A prospective multicenter cross-sectional study in patients with SpA and PsA with active disease (defined as patients who were going to start or switch biological therapy according to physician criteria and in agreement with clinical guidelines) was undertaken. Basal assessment included clinical features, physical examination and laboratory tests. Patients underwent bilateral US examination of peripheral entheses according to the MAdrid Sonographic Enthesitis Index (MASEI). MASEI and Outcome Measures in Rheumatology (OMERACT) enthesitis Power Doppler (PD) definitions were checked. Each enthesis was scanned in two planes: longitudinal and transverse, and 5 second videos were recorded for reliability. An inter-reader analysis by three readers was performed at each included center. For statistical analysis Mann-WhitneyU and Kruskal-Wallis tests were used. Intraclass correlation coefficient (ICC) and kappa test were used for reliability

Results: 64 consecutive patients were included, of whom 19(29.7\%) were ankylosing spondylitis (AS), $7(10.9 \%)$, non-radiographic axial spondyloarthritis (nr-axSpA) and $38(59.4 \%)$ PsA patients. Mean age was $52.4 \pm 12.5$ years and $36(56.3 \%)$ were males. Mean DAS28 $(3.6 \pm 1.3)$ for peripheral involvement, mean BASDAI $(5.6 \pm 2.2)$ for axial involvement, and CRP values $(10 \pm 10.9)$ reflect moderate-high disease activity at baseline. Demographic, clinical and MASEI baseline characteristics are shown in Table 1. Mean global MASEI score was $29.4( \pm 11.4)$ and 55 patients $(86 \%)$ scored $\geq 18$ (proposed cut-off point to diagnose SpA). At the patient level, abnormal US findings consistent with at least one enthesis showing PD signal were observed in 52(81.3\%) of patients using MASEI PD and $48(75 \%)$ using OMERACT PD definition without significant variation among the different $\mathrm{SpA}$ subtypes ( $p=0.8$ and $p=0.6$, respectively). The inter-reader reliability among the two cohorts from each center performed by three readers was high (ICC cohort 1:0.92; cohort 2:0.85) and inter three readers kappa was good (0.92 and 0.86 for Doppler MASEI and Doppler OMERACT respectively).

Table 1. Baseline characteristics of SpA and PsA patients

\begin{tabular}{lccccc}
\hline & $\begin{array}{c}\text { Total } \\
\mathrm{n}=64\end{array}$ & $\begin{array}{c}\mathrm{AS} \\
\mathrm{n}=19 \\
(29.7 \%)\end{array}$ & $\begin{array}{c}\text { PsA } \\
\mathrm{n}=38 \\
(59.4 \%)\end{array}$ & $\begin{array}{c}\mathrm{nr}-\mathrm{axSpA} \\
\mathrm{n}=7 \\
(10.9 \%)\end{array}$ & $\mathrm{p}$ \\
& & $(1.9 \%)$ & \\
\hline Age & $52.4 \pm 12.5$ & $50.3 \pm 14.5$ & $54.6 \pm 11.6$ & $46.3 \pm 9.9$ & 0.2 \\
Sex (Male) & $36(56.3 \%)$ & $10(52.6 \%)$ & $23(60.5 \%)$ & $3(42.9 \%)$ & 0.6 \\
CRP $(\mathrm{mg} / \mathrm{L})$ & $10 \pm 10.9$ & $13.7 \pm 11.4$ & $9 \pm 10.9$ & $6.8 \pm 9.1$ & 0.3 \\
VSG $(\mathrm{mm} / \mathrm{h})$ & $17.3 \pm 15$ & $12.6 \pm 7.5$ & $20.6 \pm 18$ & $11.9 \pm 4$ & 0.4 \\
DAS28 $\mathrm{n}=40$ & $3.6 \pm 1.3$ & $3.1 \pm 1.1$ & $3.9 \pm 1.3$ & $3.2 \pm 1.4$ & 0.2 \\
BASDAI $\mathrm{n}=23$ & $5.6 \pm 2.2$ & $5.3 \pm 2.5$ & $5.4 \pm 0.8$ & $6.9 \pm 0.9$ & 0.2 \\
MASES $\mathrm{n}=26$ & $1.1 \pm 1.5$ & $1.1 \pm 1.6$ & - & $1.1 \pm 1.3$ & 0.9 \\
MASEI & $29.4 \pm 11.4$ & $29.1 \pm 9$ & $30 \pm 12.8$ & $26.7 \pm 10.4$ & 0.9 \\
MASEI score $\geq 18$ & $55(85.9 \%)$ & $18(94.7 \%)$ & $32(84.2 \%)$ & $5(71.4 \%)$ & 0.3 \\
Mean number of enthesis with PD & $1.6 \pm 1.4$ & $1.7 \pm 1.3$ & $1.5 \pm 1.5$ & $1.6 \pm 1.7$ & 0.6 \\
$\quad$ OMERACT & & & & & \\
Mean number enthesis with PD & $2.1 \pm 1.7$ & $1.9 \pm 1.4$ & $2.2 \pm 1.8$ & $1.7 \pm 1.7$ & 0.8 \\
$\quad$ MASEI & & & & & \\
PD OMERACT $\geq 1$ & $48(75 \%)$ & $15(78.9 \%)$ & $28(73.7 \%)$ & $5(71.4 \%)$ & 0.9 \\
PD MASEl $\geq 1$ & $52(81.3 \%)$ & $15(78.9 \%)$ & $32(84.2 \%)$ & $5(71.4 \%)$ & 0.7
\end{tabular}

Conclusion: PD enthesitis is found in the vast majority of patients with active SpA and PsA, independent of SpA subtype. MASEI PD might have some advantages versus OMERACT PD definition to detect active enthesitis. These findings support the usefulness of PD US in the assessment of activity in SpA and PsA at patient level.

Disclosure of Interests: Juan Molina Collada: None declared, Cristina MacíaVilla: None declared, Chamaida Plasencia: None declared, Jose-Maria Alvaro-Gracia Grant/research support from: Abbvie, Elli-Lilly, MSD, Novartis, Pfizer, Consultant of: Abbvie, BMS, Janssen-Cilag, Elli-Lilly, MSD, Novartis, Pfizer, Sanofi, Tigenix, Roche, UCB, Paid instructor for: Elli-Lilly, Pfizer, Roche, Speakers bureau: Abbvie, BMS, Janssen-Cilag, Elli-Lilly, Gedeon Richter, MSD, Novartis, Pfizer, Sanofi, Tigenix, Roche, UCB, Eugenio de Miguel Grant/research support from: Yes (Abbvie, Novartis, Pfizer), Consultant of: Yes (Abbvie, Novartis, Pfizer), Paid instructor for: yes (AbbVie, Novartis, Pfizer, MSD, BMS, UCB Roche, Grunental, Janssen, Sanofi), Speakers bureau: yes (AbbVie, Novartis, Pfizer, MSD, BMS, UCB, Roche, Grunental, Janssen, Sanofi) DOI: 10.1136/annrheumdis-2020-eular.4867 